1 Fundação Oswaldo Cruz (Fiocruz), Escola Nacional de Saúde Pública Sérgio Arouca (Ensp), Centro

de Estudos, Políticas e Informações sobre Determinantes Sociais da Saúde (Cepi-DSS) - Rio de Janeiro (RJ), Brasil.

borde.elis@gmail.com

2 Universidad Nacional de Colombia - Bogotá (DC)

Colombia.

mehernandeza@unal.

edu.co

${ }^{3}$ Fundação Oswaldo Cruz (Fiocruz), Escola Nacional de Saúde Pública Sérgio Arouca (Ensp) - Rio de Janeiro (RJ), Brasil. marcelo.firpo@ensp. fiocruz.br

\section{Uma análise crítica da abordagem dos Determinantes Sociais da Saúde a partir da medicina social e saúde coletiva latino-americana}

\author{
A critical analysis of the social determinants of health approach from the \\ perspective of the latin american social medicine and collective health
}

\author{
Elis Borde', Mario Hernández-Álvarez ${ }^{\mathbf{2}}$, Marcelo Firpo de Souza Porto $\mathbf{3}$
}

\begin{abstract}
RESUMO O enfoque dos Determinantes Sociais da Saúde tem tido ampla difusão e foi aparentemente bem acolhido globalmente. Este artigo assume uma visão crítica desse enfoque, buscando sistematizar as críticas principalmente provenientes de debates no interior da medicina social e saúde coletiva latino-americana, que se articularam ao redor da diferenciação entre Determinantes Sociais da Saúde e a determinação social dos processos saúde-doença. Pretendemos examinar estas diferenças para problematizar a aparente unanimidade retórica em prol da equidade. Ainda que a abordagem dos Determinantes Sociais da Saúde marque um enorme avanço na mobilização pela equidade em saúde, em nossa avaliação ela não consegue ser mais do que um avanço incompleto.
\end{abstract}

PALAVRAS-CHAVE Desigualdades em saúde; Equidade em saúde; Medicina social.

ABSTRACT This article seeks to critically analyze the Social Determinants of Health approach and proposes a synthesis of the critique that has shaped the debate and particularly found expression in the differentiation between SDH and the Latin American Social Medicine and Collective Health 'social determination of the health-disease processes' approach. Reexamining the apparent rhetoric unanimity that defined the agenda of the mobilization around the Social Determinants of Health, we seek to address the differences between these approaches and conclude that the Social Determinants of Health approach marks an important but incomplete advance in the mobilization towards the reduction of health inequities.

KEYWORDS Inequalities in health; Equity in health; Social medicine. 


\section{Introdução}

Atualmente há um consenso sobre a importância de abordar os efeitos da dimensão social na saúde (BARATA; ALMEIDA-FILHO; BARRETO, 2012) e, também graças à mobilização ao redor dos Determinantes Sociais da Saúde (DSS), iniquidades sociais em saúde são reconhecidas como desigualdades sistemáticas, evitáveis, injustas e desnecessárias (CDSS, 2008). A crescente referência à importância da dimensão social na compreensão e atuação sobre as iniquidades sociais em saúde, no entanto, não implicou em uma superação dos problemas relacionados às falácias da indefinição da natureza dos processos sociais envolvidos na determinação social dos processos saúde-doença, apontadas por diversos autores desde os anos 1960, incluindo Juan Cesar García (1994). A compreensão destes processos continua sendo limitada, as intervenções para combater as iniquidades sociais em saúde são insuficientes e continuam predominando narrativas biologicistas e culturalistas nas abordagens dominantes no campo da saúde pública e, mais especificamente, da epidemiologia. Parecemos enfrentar uma situação inclusive mais complexa, devido à apropriação funcional das propostas e da terminologia crítica, culminando em uma aparente unanimidade retórica, na invisibilização de abordagens alternativas e, em consequência da última, a constituição de uma "universalidade radicalmente excludente" (LANDER, 2005, p. 26) dentro do campo teórico da epidemiologia.

Buscamos, neste artigo, analisar a abordagem das iniquidades sociais no modelo dos DSS que vem sendo promovida pela Comissão dos Determinantes Sociais da Saúde (CDSS) da Organização Mundial da Saúde (OMS) desde o ano 2005. Com a publicação do relatório final no ano 2008, o trabalho da Comissão consolidou-se como referência central na matéria de desigualdades e iniquidades sociais em saúde no mundo inteiro. No momento em que se realizou a
Conferência Mundial dos DSS (WCSDH) no Rio de Janeiro (19-21.10.2011), a proposta da CDSS se apresentou como nova agenda de pesquisa em contraposição às abordagens ainda hegemônicas na epidemiologia, que diante a dificuldade de abordar 'o social' têm optado por uma negação 'do social' e tem reforçado uma redução da epidemiologia à sua componente biomédica, artificialmente isolando os processos saúde-doença dos processos sociais mais amplos e, principalmente, das relações de poder. No entanto, o fato de surgirem três declarações alternativas à Declaração Política do Rio sobre DSS manifestou certa insatisfação e diferenças no que concerne a forma de entender a interação entre processos sociais e o processo saúde-doença e os DSS. As declarações emitidas, respectivamente, pelo Movimento para a Saúde dos Povos (People's Health Movement - $P H M$ ), a Associação Latino-Americana de Medicina Social (Alames) e o Centro Brasileiro de Estudos de Saúde (Cebes) e a Federação Internacional das Associações de Estudantes de Medicina (IFMSA) visibilizaram a existência de propostas alternativas à proposta da CDSS.

As principais diferenças articulam-se ao redor da diferenciação aparentemente sutil entre 'Determinantes Sociais da Saúde' (DSS) e 'Determinação Social dos processos saúde-doença' (DS), que é referida na declaração alternativa emitida pela Alames e pelo Cebes e veio marcar o debate, especialmente na América Latina. Enquanto a abordagem da 'Determinação Social', principalmente articulada desde a Medicina Social-Saúde Coletiva latino-americana (MS-SC), compartilha o reconhecimento da importância de abordar os efeitos da dimensão social na saúde e das iniquidades sociais em saúde como desigualdades sistemáticas, evitáveis, injustas e desnecessárias. Apesar dessa semelhança com a abordagem dos DSS, existem significativas diferenças na interpretação dos processos sociais que subjazem e engendram estas dinâmicas sistemáticas, evitáveis e injustas. 
Buscando problematizar a aparente unanimidade retórica em prol da equidade e a 'universalidade radicalmente excludente' que veio pautar a agenda científica e política no contexto da mobilização ao redor dos DSS e que incorporou uma noção de universalidade a partir de propostas teórico-políticas claramente inscritas na Medicina Social Europeia (MSE), pretendemos examinar e explicitar estas diferenças. Isso principalmente porque argumentamos que o trabalho da CDSS, em grande medida, revigorou a invisibilização de alternativas, reforçando uma insidiosa unanimidade retórica que tem impedido averiguar a intencionalidade política das propostas, podendo

deixar-nos desatentos e desarmados frente à possibilidade de se despolitizar a questão da saúde mediante a mera constatação distanciada da existência, quase naturalizada, de disparidades na ocorrência de doenças e eventos relativos à saúde. (ALMEIDA-FILHO, 2009, P. 368).

Entendemos que a mobilização global em torno dos DSS parecia requerer certa unidade, ao menos aparente, e uma certa visão universal para convocar à "redução das desigualdades no período de uma geração" (CDSS, 2008, P. 1). Mas parece-nos importante situar as propostas da CDSS e explorar as diferenças que se evidenciam na comparação com propostas articuladas desde a MS-SC, indicando porque a abordagem dos DSS continua sendo insuficiente para compreender e contribuir para a transformação das iniquidades sociais em saúde.

Seguindo a introdução da proposta dos DSS na primeira parte do artigo, propomos uma sistematização das críticas articuladas ao redor da abordagem dos DSS, enfatizando as diferenças que fazem a diferença na abordagem das iniquidades sociais em saúde e se expressam nos planos ontológico, epistemológico, ético e político, principalmente no que concerne aos critérios que orientam o julgamento das iniquidades e injustiças em saúde, o tipo de ação política previsto e os processos e sujeitos sociais da mudança na abordagem dos DSS e da DS, respetivamente.

\section{Os Determinantes Sociais da Saúde segundo a CDSS}

Depois do retrocesso que significou a substituição da concepção original de Atenção Primária à Saúde por 'pacotes mínimos' focalizados no marco da expansão de agendas neoliberais nos anos 1980 que também vieram a definir a agenda da OMS, a formação da CDSS no ano 2005 marcou uma importante cisão e tem sido motivo de regozijo e esperança (TAMBELLINI; SCHÜTZ, 2009; NOGUEIRA, 2010; INGLEBY, 2012; CABRERA ET AL., 2011). Cabrera et al. (2011) e Breilh (2011) alegam que o trabalho da Comissão tem contribuído para a denúncia das iniquidades em saúde e tem dado visibilidade mundial aos determinantes sociais, econômicos, políticos, culturais e ambientais da saúde que se configuram no marco das injustiças sociais. Para Birn (2009), a conformação da CDSS constitui um triunfo contra o paradigma dominante, que entende a saúde como instrumento e impulsor do crescimento econômico ao invés de um direito e valor humano intrínseco. O paradigma dominante sistematicamente tem negado a dimensão social das desigualdades em saúde, privilegiando abordagens biologicistas, culturalistas e, principalmente, aquelas que têm explicado as desigualdades em saúde como efeitos de decisões pessoais e estilos de vida pouco saudáveis. Birn (2009) aponta três grandes contribuições da CDSS: a) proporcionar maior legitimidade aos determinantes sociais, b) discutir as dimensões mundiais das iniquidades em saúde e c) identificar o papel dos sistemas de serviços de saúde como determinantes da saúde.

Em termos gerais, a CDSS propôs um modelo segundo o qual existem dois tipos de Determinantes Sociais da Saúde: os determinantes estruturais e os determinantes 
intermediários. Os determinantes estruturais terão impacto na equidade em saúde e no bem-estar através da sua ação sobre os determinantes intermediários. Daí a diferenciação entre 'determinantes estruturais das iniquidades em saúde' e 'determinantes intermediários da saúde', que em conjunto constituem os 'Determinantes Sociais da Saúde' na proposta da CDSS, que veio a se consolidar principalmente no marco conceitual (SOLAR; IRWIN, 2010) e no relatório final (CDSS, 2008).

\section{Determinantes estruturais das iniquidades sociais em saúde}

Os determinantes estruturais são entendidos como motores estruturais das condições de vida e se aproximam ao conceito de determinantes distais, ou macrodeterminantes econômicos e sociais, segundo o conhecido modelo de Whitehead e Dahlgren (1991). Solar e Irwin (2010) apontam no marco conceitual da CDSS, que os determinantes estruturais são os mecanismos que geram e reforçam hierarquias sociais que definem o poder, prestigio e acesso a recursos por meio da educação, do trabalho e da renda e, desta forma, configuram circunstâncias materiais (condições de vida e de trabalho etc.), fatores comportamentais e biológicos, coesão social e fatores psicossociais - os determinantes intermediários da saúde que completam os Determinantes Sociais da Saúde. Entre os mecanismos que produzem e reforçam a hierarquia social, os autores destacam os seguintes fatores contextuais (SOLAR; IRWIN, 2010): 1) cultura e valores sociais, 2) políticas públicas em áreas como educação, atenção médica, água e saneamento, 3) políticas sociais afetando fatores como emprego, bem- estar social, posse de terra e vivenda, 4) políticas macroeconômicas, incluindo políticas fiscais, monetárias, políticas de comércio e a estrutura do mercado laboral, 5) governança em relação aos mecanismos de participação social da sociedade civil, prestação de contas e transparência na administração pública e padrões de discriminação e 6) condições epidemiológicas, principalmente no caso de epidemias que alteram a estrutura social como, por exemplo, a epidemia do HIV/Aids em algumas partes do mundo.

\section{Determinantes intermediários da saúde}

Os determinantes intermediários referem ao conjunto de elementos categorizados em fatores comportamentais e fatores biológicos, circunstâncias materiais (condições de vida e de trabalho, disponibilidade de alimentos, moradia etc.), e fatores psicossociais. Ainda incluem o sistema de saúde e a coesão social. Além da vulnerabilidade diferenciada, destacam-se diferenças na exposição e nas consequências de acordo à posição socioeconômica. Para Solar e Irwin (2010), as circunstâncias materiais são os fatores intermediários mais importantes e referem, entre outros, às condições de moradia, particularmente a superlotação, a qualidade de ar, o acesso à agua e as caraterísticas da vizinhança. Em relação às condições de trabalho, os autores afirmam que existem marcadas diferenças sociais no que concerne a exposição a agressores físicos, mentais, químicos e ergonômicos no local de trabalho e ainda destacam a vulnerabilidade diferenciada de trabalhadores em economias informais. Outros determinantes intermediários da saúde propostos por Solar e Irwin (2010) e contidos no modelo proposto no marco conceitual da CDSS (figura 1), são os estilos de vida e comportamentos, que se expressam, entre outros, nos padrões de consumo de tabaco, álcool e na falta de atividade física. Os dados revisados por Solar \& Irwin (2010) sugerem que os fatores de risco operam de forma diferencial de acordo à posição socioeconômica. 
Incluem-se ainda estressores psicossociais (negative life events and job strain), circunstâncias estressantes (por exemplo, dívidas) e a falta de apoio social como determinantes intermediários da saúde. No marco conceitual da CDSS, o sistema de saúde também é considerado um determinante intermediário da saúde, reconhecendo principalmente a influência das barreiras de acesso. A coesão social é proposta como determinante transcendental e é entendida como uma forma de dar conta do impacto das relações estabelecidas entre as comunidades e instituições, particularmente o Estado, sobre a qualidade de vida das comunidades (SOLAR; IRWIN, 2010).

Figura 1. Modelo proposto no marco conceitual da CDSS

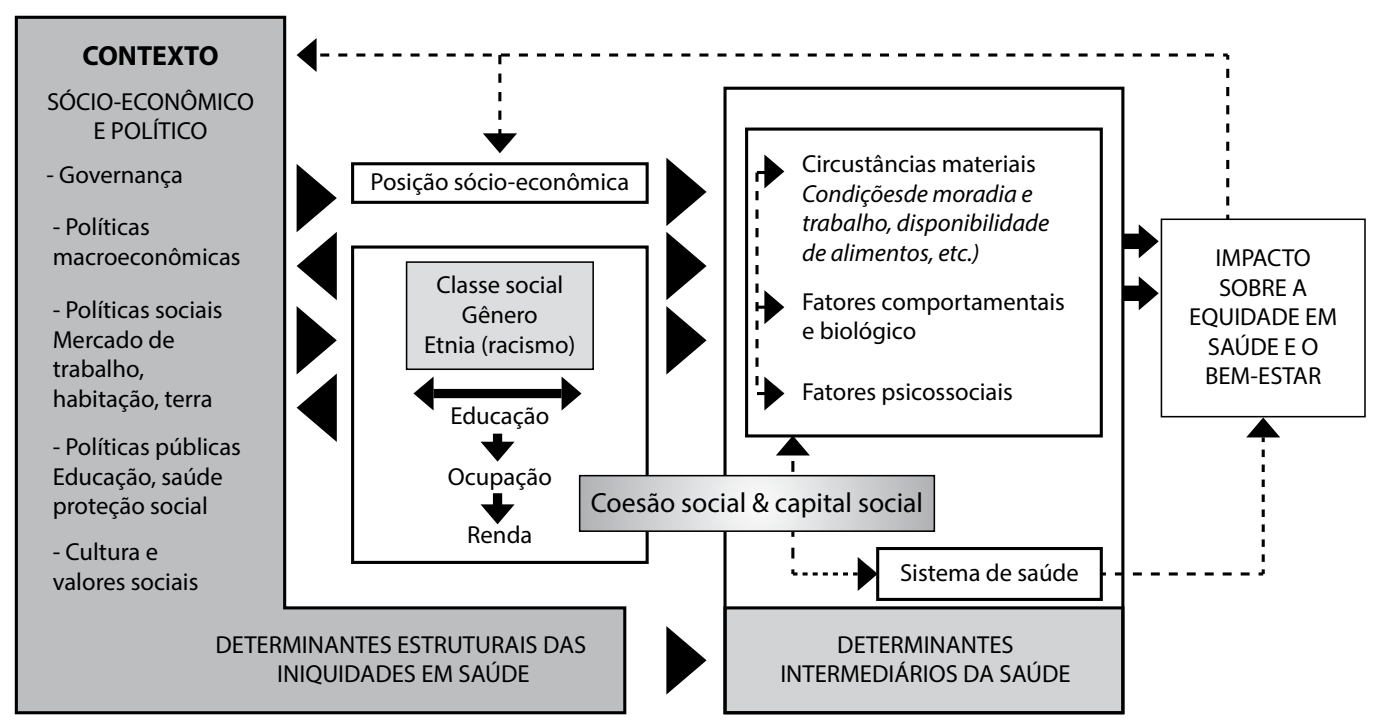

Fonte: SOLAR; IRWIN, 2010

Como foi referido no início desta seção, a abordagem dos DSS e o lançamento do relatório final da CDSS podem ser considerados como avanços na mobilização internacional em prol da equidade em saúde. A proposta da CDSS articulada no relatório final da CDSS (2008) e no marco conceitual (SOLAR; IRWIN, 2010), parte do reconhecimento de que

a saúde deficiente dos pobres, a gradação social na saúde dentro dos países e as desigualdades profundas entre países são provocadas pela distribuição desigual de poder, rendimentos, bens e serviços, nas escalas nacionais e global. (CDSS, 2008, P. 1).
Dessa forma, traz denúncias que marcam uma importante cisão com respeito aos relatórios anteriores da OMS como, por exemplo, o polêmico 'Relatório Mundial da Saúde para o Ano 2000'. Ainda desafia a corrente hegemônica no campo da saúde pública, que continua ditando as reformas no setor saúde e se define por uma posição liberal individualista de justiça e uma compreensão da saúde aprisionada à visão biomédica (HERNÁNDEZÁlvAREZ, 2011). Sem dúvida a CDSS propõe uma crítica importante quando reconhece que as assimetrias de poder são geradas e reproduzidas pelo contexto socioeconômico e político, que privilegia grupos diferenciáveis 
por classe social, gênero, etnia, educação, ocupação e renda (posição socioeconômica), configurando padrões diferenciados de vulnerabilidade e exposição aos determinantes intermediários de saúde e, desta forma, definem as iniquidades sociais em saúde. Porém tal crítica é incompleta por conceber no atual modelo de sociedade a possibilidade de reverter as iniquidades.

A proposta da CDSS, neste sentido, se concentra no que produz a sociedade em termos de desigualdades e reduz a margem de compreensão e de denúncia aos fatores mensuráveis e claramente visíveis, esperando que isso, por si só, seja suficiente para reverter o problema. Ou seja, não propõe uma análise compreensiva das relações de poder e dos padrões de exploração, dominação e marginalização que subjazem as hierarquias sociais. Classe social, etnia e gênero não aparecem como categorias analíticas da matriz de dominação que tem sustentado a consolidação e expansão do sistema-mundo capitalista moderno/ colonial. Ao contrário, parte-se de uma visão a-histórica e abstrata que baseia as categorias analíticas no que é empiricamente observável e não explicita porque as hierarquias sociais têm-se configurado em função e em relação ao gênero, à classe social, à raça e etnia, e qual o papel da educação, da ocupação e da renda nestas relações. Por isso, a crítica formulada, paradoxalmente, ao mesmo tempo em que avança, imobiliza.

É talvez este o ponto central da crítica que se articulou em relação à CDSS e à abordagem dos DSS, que culminou em publicações científicas (PASSOS, 2009; EIBENSCHUTZ; TAMEZ; GONZÁLEZ， 2011; INGLEBY, 2012; NOGUEIRA, 2010; PEÑARANDA; OTÁLVARO, 2013) e em declarações políticas, como foi referido acima. Cabe abordar com mais detalhe os diferentes pontos de crítica que foram articulados, principalmente desde a MS-SC, que hoje talvez seja a corrente contra hegemônica mais importante no campo da saúde pública.

\section{Diferenças que fazem a diferença: pontos de crítica}

As críticas articuladas em relação à abordagem dos DSS apontam insuficiências no alcance das análises e nas propostas articuladas pela CDSS, e alguns autores (BIRN, 2009; INGLEBY, 2012) referem ao relatório final da CDSS como uma 'oportunidade perdida'. Referemse, neste sentido, à falta de consideração de processos considerados centrais na produção e reprodução das iniquidades sociais em saúde como, por exemplo, a migração (INGLEBY, 2012). De acordo com o resumo das críticas mencionadas na seção anterior, ainda se questiona a abordagem histórica e descontextualizada das iniquidades sociais enquanto efeitos de um 'contexto socioeconômico e político' abstrato, que evidencia a persistência de lacunas, omissões e desinteresses na abordagem das iniquidades sociais da saúde desde a perspectiva da CDSS (ALMEIDA-FILHO, 2009). Vicente Navarro (2009, P. 440), neste sentido, afirma:

The Commission's report goes very far in describing how inequalities are killing people [...] And we, as public health workers, must denounce not only the process, but the forces that do the killing.

Estes problemas se manifestam de forma particularmente clara em relação à abordagem da saúde implícita na proposta da CDSS e na abordagem do 'social' dos determinantes da saúde, incluindo as noções de justiça que são implicadas. A seguir pretendemos aprofundar estes pontos de crítica e abordar as diferenças que se evidenciam na comparação com propostas articuladas desde a MS-SC, buscando ampliar e complementar análises feitas por Morales et al. (2013), Eibenschutz, Tamez e Gozáles (2011) e Peñaranda e Otálvaro (2013), entre outros. 


\section{Saúde: entre estado de ausência de doença ou processo social}

A teoria da saúde adotada no relatório final da CDSS e no marco conceitual coincide com a teoria que impera na medicina ocidental e também com a concepção assumida pela epidemiologia dominante. Neste sentido, Nogueira (2010) aponta que na proposta da CDSS, a saúde equivale a ausência de morbimortalidade e a sobrevivência física no longo prazo (longevidade). Para o autor a saúde é definida exclusivamente a partir das condições de normalidade e anormalidade psico-biológicas dos indivíduos, estabelecidas pela biomedicina. Trata-se, segundo Hernández-Álvarez (2011), de uma concepção tradicional da saúde como equilíbrio biológico ou energético-material, de expressão individual e com causas internas e externas empiricamente identificáveis como 'fatores' - no caso, 'determinantes'. Desta forma se diferencia da noção do 'processo saúde-doença', articulada pela MS-SC, como processo de natureza social com expressão específica no 'nexo biopsíquico humano' e 'subsumido' nos processos de produção e reprodução social (LAURELL, 1987; BREILH, 2003).

A abordagem da CDSS desconsidera estas dimensões, principalmente a complexidade, multidimensionalidade e natureza social do processo saúde-doença, que vão muito além do reconhecimento da influência de fatores sociais sobre a saúde das populações (NOGUEIRA, 2010). Ao contrário, a 'determinação social dos processos saúde-doença' implica reconhecer as dinâmicas sociais que engendram os processos de produção e reprodução dos padrões estruturais de dominação, exploração e marginalização nas sociedades concretas, moldam os modos de vida e se expressam nos processos saúde-doença.

\section{A abordagem do social}

Na abordagem dos DSS, a organização social que se configura a partir destes processos é concebida como externa aos indivíduos e grupos sociais (CABRERA ET AL. 2011) e desta forma, as variáveis do 'contexto socioeconômico e político' que sugere a CDSS são definidas apenas em termos abstratos. Por mais que se reconheça o papel do mercado e da globalização na geração das iniquidades sociais e se demande uma 'participação equitativa' no comércio internacional e nas instituições econômicas mundiais (BIRN, 2009), a não-explicitação do tipo de sociedade e de economia nos quais se desenvolvem estas relações injustas e para os quais são funcionais, insinua uma abordagem abstrata, superficial e inerentemente limitada dos processos que engendram as iniquidades sociais em saúde. O mercado e a globalização assim aparecem como 'forças obscuras' e abstratas que apresentam 'falhas' na medida em que produzem desigualdades em educação, ocupação e renda (CABRERA ET AL., 2011). Tais falhas ou externalidades poderiam ser corrigidas na medida em que se adotam boas práticas na governança, políticas redistributivas e o mercado se torne mais 'responsável' (CDSS, 2008). A CDSS, neste sentido, sugere aumentar a representação de defensores pela equidade em saúde e apoiar a institucionalização da avaliação do impacto sobre a equidade sanitária (BIRN, 2009). Ambas as propostas certamente são interessantes e pragmáticas, mas claramente insuficientes e bastante ingênuas, delegando para os 'especialistas do direito' a responsabilidade para direcionar a sociedade rumo a modelos mais justos. Os impactos negativos que gera 'o mercado' sobre a equidade em saúde, desta forma, são considerados como aleatórios, acidentais e não-desejados, e o padrão sistemático da determinação social que se engendra, entre outros, a partir da superexploração do trabalho, da natureza implicada no regime de acumulação capitalista e das diferentes formas 
de discriminação e racismo, não é reconhecido pela CDSS. Também não se reconhece a incompatibilidade do regime de acumulação capitalista com modos de vida saudáveis, como é colocado por Breilh (2013). A CDSS, portanto, se contenta com melhorias num sistema inerentemente injusto (BREILH, 2011) e, desta forma, segue uma abordagem característica da sociologia funcionalista, na medida em que estorva um pensamento alternativo sobre possíveis alternativas, principalmente ao regime de acumulação capitalista. $\mathrm{O}$ horizonte de transformação da CDSS se limita à correção das distorções que acompanharam a aceleração e extensão do regime de acumulação capitalista, sem ameaçá-lo (BREILH, 2011), e reserva um papel estratégico à gestão pública redistributiva. Sem dúvida esta abre espaços importantes, que, no entanto, são insuficientes para uma transformação mais profunda.

Da mesma forma pode-se dizer que a insistência nas políticas redistributivas certamente é um passo fundamental na mobilização pela equidade em saúde no contexto das políticas de austeridade e no contexto da expansão da influência de agendas neoliberais no mundo inteiro. Navarro (2009), no entanto, faz uma importante observação ao notar que os chamados da CDSS para políticas de redistribuição de recursos não explicitam quem concentra os recursos e porquê. Cabe retomar Nogueira (2010, P. 405) ao apontar que

essa nova epidemiologia, que é apenas pseudossocial, arroga a si o direito de falar do social sem compreendê-lo como tal, sem ser capaz de adotar a este respeito qualquer abordagem teórica razoável, e muito menos, uma teoria crítica da sociedade.

Os determinantes sociais e condicionantes dos efeitos observados em relação às desigualdades sociais em saúde apenas são colocados como fatores e/ou situações sociais que as precedem, o que Tambellini e Schütz (2009) relacionam à falta de precisão conceitual e à teorização frouxa sobre a relação saúde-sociedade que se manifesta na proposta da CDSS. Esta adota um conceito estreito de determinantes sociais que opera num nível da empiria, insinuando uma compreensão do social como algo que se encontra nas populações e influencia a natureza essencialmente biológica do homem (NOGUEIRA, 2010). Reforça, ainda, uma visão fragmentada da realidade na medida em que repete o modus operandi do causalismo (BREILH, 2011), enxerga e atua apenas sobre fatores, e não sobre a mudança de processos estruturais, como foi referido em relação ao horizonte de transformação. Segundo Breilh (2011), esta abordagem impossibilita o estabelecimento de um nexo histórico real entre os determinantes estruturais e os determinantes intermediários da saúde, sobretudo porque as categorias definidoras do conjunto e da lógica dos determinantes estruturais estão ausentes. Por exemplo na conceituação da classe social evidencia-se um foco distorcido para a dimensão do consumo (educação - ocupação - renda), que evidentemente é insuficiente para dar conta das dinâmicas de marginalização, dominação e exploração que produzem e reproduzem as iniquidades sociais. Almeida-Filho (2009), neste sentido, observa que os conceitos dominantes de desigualdades em saúde manifestam uma 'falácia econocêntrica', o qual também se aplica à proposta dos DSS. Renda, produção, consumo e outros indicadores econômicos são tomados como parâmetros para medidas de desigualdade na sociedade, desprezando o papel de outras dimensões de subalternização que podem, inclusive, ter maiores impactos sobre o processo saúde-doença e sobre o bem-estar coletivo, principalmente em sociedades que se afastam do mítico pleno emprego e são ditadas por economias informais e não capitalistas. 
A falácia econocêntrica tem marcado as interpretações dominantes das iniquidades sociais e na proposta dos DSS é reforçada pela ênfase no 'gradiente em saúde', que apenas se aplica às variáveis contínuas (por exemplo: renda) e não pode ser aplicada às variáveis ordinais (por exemplo: raça/etnia, gênero, classe social), como apontado por Ingleby (2012). O mesmo autor reconhece a importância de optar pela ênfase no 'gradiente' para examinar toda a gama de desigualdades e superar as dicotomias simplicistas, mas critica que esta ênfase levou à desconsideração das 'brechas' (gaps) entre grupos socialmente construídos, em relação e em função dos quais historicamente se geraram iniquidades.

\section{Diferenças na abordagem da justiça, injustiça, equidade $e$ iniquidade}

O conceito mais destacado para abordar as desigualdades em saúde na abordagem dos DSS é a iniquidade, entendida como desigualdade injusta. Cabe, portanto, indagar, mesmo de forma sucinta, sobre as noções de justiça implicadas a esse conceito. Partimos, neste sentido, do reconhecimento da iniquidade como conceito ético que implica uma valorização da desigualdade.

A proposta dos DSS assenta-se numa posição liberal redistributiva da justiça influenciada por John Rawls e Amartya Sen (SOLAR; IRWIN, 2010), que marca a maneira de entender a causalidade das iniquidades sociais e, desta forma, configura as respostas políticas e o âmbito privilegiado para as transformações. De acordo com Hernández-Álvarez (2011), cabe diferenciar a posição de justiça adotada pela CDSS de principalmente duas posições alternativas: a corrente hegemônica liberal da justiça e, por outro lado, a posição igualitarista social, que tem marcado as propostas da MS-SC.

A posição liberal da justiça tem sido hegemônica nas reformas das políticas sociais e sistemas de proteção nos últimos 30 anos no mundo inteiro (HERNÁNDEZ-ÁLVAREZ, 2003) e tem-se guiado por uma noção de justiça que assume como justas aquelas transações que são feitas entre indivíduos livres e iguais (HERNÁNDEZÁlVAREZ, 2011). Como é colocado por HernándezÁlvarez (2011), o ponto central da posição liberal da justiça consiste em que os direitos sociais, econômicos e culturais não são estritamente direitos, mas conformam bens e serviços associados à capacidade de pagar: "Quien tiene, paga y obtiene. Quien no tiene, debe demostrarlo para merecer una atención subsidiada y focalizada por parte del Estado" (HERNÁNDEZ-ÁLVAREZ, 2011, P. 172). A perspectiva liberal da justiça, em sua visão individualista e utilitarista, dialoga bem com o assim chamado 'individualismo metodológico', que assume a sociedade como um conjunto de agentes racionais, que se encontra em transações egoístas e procura maximizar a utilidade (HERNÁNDEZ-ÁlVAREZ, 2011). Hernández-Álvarez aponta que as políticas baseadas nesta perspectiva de justiça têm resultado politicamente viáveis na medida em que geram melhoras de curto prazo, sem a necessidade de modificar as condições que geram as iniquidades e a pobreza.

A CDSS se distancia desta posição de justiça hegemônica ao reconhecer estruturas sociais que engendram desigualdades na 'liberdade de' ser e na 'liberdade para' fazer, principalmente através da sua influência sobre escolaridade, ocupação e renda. Reconhece que existem diferenças no grau de liberdade e igualdade dos homens, que devem ser equilibradas a partir da garantia de condições de vida digna, expressadas em trabalho, educação, saneamento, assistência médica e moradia para todos os cidadãos. A iniquidade, portanto, é entendida como injustiça no repartir e no acesso às riquezas e ao poder, com a estratificação social sendo concebida como resultado de desigualdades em relação à escolaridade, ocupação e renda que surgem de contextos sociopolíticos desfavoráveis. Desigualdades em saúde são concebidas como injustas quando são produto de situações que estão fora do controle dos indivíduos e limitam o exercício da 'liberdade 
individual' (agência) reconhecendo que, de acordo à posição socioeconômica - entendida como produto da interação entre o nível de educação, o tipo de ocupação, a renda, o gênero, a etnia/exposição ao racismo e a classe social -, o grau de escolha de estilos de vida varia. A CDSS refere, entre outros, a alimentação restringida por carências econômicas, a exposição a condições de vida e trabalho insalubres, a violação dos direitos humanos e o acesso inadequado a serviços básicos como saneamento e moradia. Nesta concepção, uma sociedade justa se caracteriza por proporcionar um elevado grau de liberdade para todos (MORALES ET AL,, 2013).

A posição igualitarista social da justiça parte do reconhecimento de que as desigualdades são injustas porque são produzidas por uma apropriação indevida dos meios de realização da vida por parte de uma classe social, um gênero e/ou uma etnia/raça (matriz de dominação) (HERnÁNDEZ-ÁlvAREZ, 2011). Reconhece desta forma um 'sujeito histórico', construído nas relações sociais e processos de dominação, exploração e marginalização em função da consolidação de privilégios (materiais, políticos e simbólicos). Desde esta perspectiva, iniquidades e desigualdades são produzidas pelas relações de poder e de dominação, que se articulam no regime de acumulação capitalista, e são confrontadas pelas resistências e lutas dos explorados pelo regime, ou ainda dos que vivem, ainda que parcialmente, em contextos não capitalistas, como algumas comunidades indígenas e camponeses. A estratificação social, portanto, é entendida como expressão das formas de propriedade, poder e divisão do trabalho características da sociedade capitalista e das suas formas de produção e reprodução. Isso implica que a iniquidade não apenas se refere às injustiças na distribuição e acesso aos bens materiais e serviços básicos, senão ao processo intrínseco que as engendra. Retomando o exemplo discutido acima, a exposição diferencial a condições de vida e trabalho insalubres não pode ser considerada aleatória, mas deve ser reconhecida como sistemática e funcional à expansão e consolidação do regime de acumulação capitalista e assente nos vários mecanismos de dominação (LANDER, 2005) e na divisão internacional do trabalho. Portanto, as hierarquias étnico-racial, de classe social e gênero são simultaneamente parte constitutiva do desenvolvimento e da reprodução do regime capitalista e colonial, bem como determinantes do grau e da qualidade de dominação. As transformações, assim, não podem se limitar ao poder estatal e à qualidade de políticas públicas, dos "gestores" e dos processos participativos, mas surgem da "intersubjetividad emancipadora entre las clases, los géneros y las etnias dominados” (BREILH, 2003, P. 60).

\section{Propostas de transformação ou como reduzir as desigualdades no período de uma geração}

As diferenças na abordagem da justiça e da equidade têm implicações para a prática, como é enfatizado por Hernández-Álvarez (2011). Cabe, portanto, problematizar as referências centrais e revisar as propostas apresentadas pela CDSS para reduzir as iniquidades sociais em saúde no período de uma geração. Em linhas gerais, a CDSS propõe ações em dois níveis (BREILH, 2011): nos condutores estruturais e nas mudanças das condições de vida. O primeiro grupo de medidas recomendadas pela CDSS inclui o financiamento justo, orientado para aumentar o gasto em saúde; as responsabilidades do mercado; a equidade de gênero; a tomada de decisões imparcial a partir de um empoderamento que garanta uma ampla participação na tomada de decisões referente à equidade em saúde e à boa condução governamental global, contando com um maior peso de organismos multilaterais que incluam os DSS nas suas agendas. O segundo grupo de medidas se dirige a alcançar: equidade desde o início da vida; emprego justo e trabalho decente, combatendo o trabalho precarizado, o desemprego 
e as más condições de trabalho; moradias saudáveis com acesso a bens e planejados para promover a saúde; acesso universal à atenção médica, promovendo sistemas universais e gratuitos, fundados na atenção primária e que garantam acesso equitativo e proteção social universal. A CDSS ainda recomenda três princípios de ação: melhorar as condições de vida cotidianas; abordar a distribuição desigual de poder, dinheiro e recursos; quantificar e compreender o problema e avaliar o impacto da ação. Na articulação dos três princípios de ação, a CDSS é inequívoca na defesa de sistemas de saúde universais e advoga pelo aumento imediato dos gastos públicos em saúde - demandas que a MS-SC também têm reivindicado. As diferenças manifestam-se principalmente nos atores e processos sociais vislumbrados para alcançar a equidade em saúde no período de uma geração desde as respetivas propostas. A CDSS imagina uma mobilização social em prol da saúde, partindo da suposição de que o reconhecimento das desigualdades em saúde sejam motivo suficiente para mobilizar autoridades e a sociedade civil. Além de ingênua, esta suposição desconhece os processos, os contextos sócio-históricos e as lutas sociais que têm permitido um avanço na mobilização em prol da equidade em saúde e se manifestam, entre outros, na consolidação do direito à saúde, na melhoria das condições de vida e trabalho, bem como o acesso mais amplo aos serviços de saúde. Cabe, neste sentido, lembrar que a reforma sanitária brasileira surgiu no contexto das conjunturas da redemocratização da sociedade e da reformulação constitucional (NOGUEIRA, 2010). Birn (2009) ainda questiona a abordagem ahistórica que a CDSS faz dos países/regiões pobres, que "atingiram um bom nível de saúde, acima de todas as expectativas, tendo em conta o nível econômico nacional” (CDSS, 2008, p. 36). A autora destaca que

en cada uno de las sociedades citadas se ha necesitado una larga lucha política, ya sea por medio de una revolución (en el caso de Cuba); participación y activismo sindical extremadamente altos (entre el 70 y el 95\% de la fuerza laboral activa, a su vez resultado de la lucha política), combinados con la elección de partidos políticos con valores socio democráticos (en los países nórdicos); una lucha larga y constante de los partidos políticos de izquierda para ser electos y reelectos (en Kerala); o movimientos populares y laborales a favor de protección social y un fin al gasto militar, después de una guerra civil brutal y de rechazo de intereses imperialistas (Costa Rica). (BIRN, 2009, P. 193).

Com a descontextualização histórica dos avanços na mobilização pela equidade em saúde e a negação do papel dos movimentos sociais a partir de um foco tecnicista na gestão pública (BIRN, 2009), a ênfase na 'participação social' e no 'empoderamento' perdem significância e aparecem como conceitos soltos, esvaziados de conteúdo crítico, principalmente porque são abordados em termos de identidade civil, liberdade e autonomia, contrário à compreensão da emancipação como superação das relações de poder existentes. De forma semelhante, o termo 'vontade política', posto no centro das recomendações da CDSS, continua sendo superficial, indefinido e, de acordo com Birn (2009), sem sentido.

Cabe, consequentemente, questionar o alcance das reformas propostas pela CDSS, que Breilh (2011) acusou de se limitar a uma forma de mascaramento social, que melhora algumas partes mas não enfrenta as bases estruturais da determinação social dos processos saúde-doença. Isso, principalmente, porque se negam trajetórias históricas de lutas e atores sociais que têm sido fundamentais na elevação do bem-estar das populações historicamente excluídas e na proteção da vida, e poderiam visibilizar horizontes alternativos desde os quais seria possível contribuir de forma decisiva para a equidade em saúde. Da mesma forma, as propostas éticas e políticas que propõe a CDSS carecem de elementos emancipatórios. Como aponta Breilh (2003), cabe notar, como demonstra a história, que avançamos pouco enfocando apenas os objetos de transformação, sem considerar os respetivos sujeitos de transformação. 
1 Nosso foco é a América Latina, mas cabe notar que a CDSS também não parece dialogar amplamente com autores asiáticos e africanos.

\section{A modo de conclusão}

Uma das contribuições atribuídas à CDSS é seu alcance global (NOGUEIRA, 2010; BIRN, 2009), dada sua pretensa capacidade de mobilizar tomadores de decisão do mundo inteiro de diferentes países e realidades sociais a partir do reconhecimento da importância de iniquidades relativas e da pobreza absoluta e suas implicações para a saúde, tanto no Norte quanto no Sul (BIRN, 2009). Mas até que ponto tal pretensão tem sido tão global? Trata-se de uma proposta globalmente relevante? Estabelece um diálogo global? Ao nosso ver, nenhuma destas perguntas pode ser respondida de forma afirmativa, pois a proposta da CDSS manifesta o que anteriormente designamos como uma 'universalidade radicalmente excludente'.

Segundo Breilh (2011), a decisão de ignorar abordagens profundamente questionadoras $\mathrm{e}$ críticas sobre as relações de poder nas sociedades não pode ser explicada por barreiras de língua. A invisibilização das contribuições da MS-SC no relatório final da CDSS e no marco conceitual, a nosso ver, parece manifestar um padrão mais sistemático enquanto mecanismo de subalternização das 'epistemologias do sul', ou seja, concepções e experiências de conhecimento alternativas às epistemologias hegemônicas eurocêntricas denominadas por Boaventura de Souza Santos de 'epistemologia da cegueira' (SANTOS, 2010). Nesse sentido, cabe notar que nem o relatório final (CDSS, 2008) nem o marco conceitual (SOLAR; IRWIN, 2010) da CDSS fazem menção aos autores latino-americanos que vêm desenvolvendo práticas, pesquisas e modelos teóricos sobre a determinação social desde os anos 1970. A CDSS quase exclusivamente se baseia na medicina e Epidemiologia Social Europeia (ESE) e dialoga com teorias desenvolvidas no Norte global e epistêmico, reduzindo a revisão da teoria sobre DSS às teorias psicossociais (CASSEL, 1976; MARMOT, WILKINSON, 2001), às teorias da produção social da doença/economia política da saúde (KAPLAN ET AL., 1996; DAVEY SMITH; EGGER, 1996; LYNCH ET AL., 2001) e à teoria ecossocial e abordagens multi-nível
(KRIEGER, 2001) (SOLAR; IRWIN, 2010). A América Latina ${ }^{1}$ apenas aparece em alguns exemplos de programas bem-sucedidos como Barrio Adentro na Venezuela (SOLAR; IRWIN, 2010) ou o Programa Saúde da Família no Brasil (CDSS, 2008), e as contribuições da MS-SC são mencionadas em menos de uma linha no marco conceitual, referenciando uma autora (TAJER, 2003) que não pode ser considerada central na produção acadêmica da MS-SC (SOLAR; IRWIN, 2010).

Como a CDSS em grande parte retoma o enfoque da ESE, não pode ser isolada da história de dissensos científicos que se tem articulado no campo da saúde pública e da epidemiologia ao longo do tempo. Breilh (2011), inclusive, reconhece a abordagem dos DSS como um novo eixo na história de dissensos, seguindo as confrontações que se deram no século XIX entre contagionismo conservador e doutrina miasmática, entre explicações unicausais e a medicina social na primeira metade do século XX, e a oposição entre modelos multicausais e o paradigma crítico. É importante notar que o problema, ao nosso ver, não consiste propriamente na adoção do enfoque da ESE, que inclusive significaria um enorme avanço no panorama atual no campo da saúde pública e epidemiologia, ainda dominado por abordagens que excluem à dimensão social e atribuem um valor apenas econômico à saúde. O problema consiste, e por isso insistimos na referência ao caráter 'radicalmente excludente', na negação desta inscrição, na invisibilização de outras abordagens e na pretensão de universalidade (LANDER, 2005). Esta pretensão se manifesta nos relatórios da CDSS, que de forma geral não fazem menção de adaptações necessárias ou de um alcance limitado do modelo, nem explicitam a inscrição teórico-conceitual e política que marca a abordagem.

De certa forma, pode-se afirmar que a criação da CDSS e a publicação do relatório final reforçou o predomínio epistemológico de abordagens provenientes do Norte global e epistêmico no campo da saúde pública e da epidemiologia, mesmo com a ESE sendo subalterna dentro do panorama atual. Neste sentido, 
criou-se, um monopólio sobre o estudo das iniquidades sociais em saúde na medida em que se invisibilizaram abordagens como, por exemplo, as da MS-SC, e se instalou o modelo dos DSS na institucionalidade internacional e nacional. Isso se reflete na referência agora quase mecânica do termo 'determinantes sociais da saúde' e 'equidade em saúde', muitas vezes desprovida até do conteúdo proporcionado pela CDSS, em propostas de pesquisa, estratégias de saúde, textos de lei e outros.

Por mais que a abordagem dos DSS marque um relevante avanço na mobilização em prol da equidade em saúde, ela não consegue superar a noção de causalidade que impera na epidemiologia convencional e, consequentemente, reforça uma noção de eventos independentes ou relacionados por meio de ligações unidirecionais, necessárias, específicas e capazes de gerar o desfecho (BARATA; ALMEIDA-FILHO; BARRETO, 2011). Desta forma, os processos saúde-doença e a determinação social das iniquidades são reduzidas a uma combinação aleatória de eventos relacionados porém discretos (políticas 'ruins', práticas discriminatórias, baixa renda etc.), reforçando uma visão descontextualizada das iniquidades sociais em saúde.

\section{Referências}

ALMEIDA-FILHO, N. Problemática teórica da determinação social da saúde (nota breve sobre desigualdades em saúde como objeto de conhecimento). Saúde em Debate, Rio de Janeiro, v. 33, n. 83, p. 349-370, 2009.

BARATA, R., ALMEIDA-FILHO, N., BARRETO, M. Epidemiologia social. In: ALMEIDA-FILHO, N.; BARRETO, M. (Org.), Epidemiologia e saúde. Fundamentos, métodos, apliações. Rio de Janeiro: Editora Guanabara Koogan, 2012.

BIRN, A. E. ¿̨Plitizándolo o puliéndolo? Subsanar las desigualdades en una generación: alcanzar la equidad sanitaria actuando sobre los determinantes sociales de la salud. Medicina Social, New York, v. 4, n. 3, p. 189-207, 2009.

BREILH, J. Epidemiologia crítica. Buenos Aires: Lugar Editorial, 2003.

La determinación social de la salud como herramienta de transformación hacia una nueva salud pública (salud colectiva). Revista Facultad Nacional de Salud Pública, Medellín, v. 31, 2013, p. 13-27.

Una perspectiva emancipadora de la investigación e incidencia basada en la determinación social de la salud. In: EIBENSCHUTZ, C., TAMEZ, S, GONZÁLEZ, R. ¿¿Determinación social o determinantes sociales de la salud? Memoria del Taller Latinoamericanos sobre Determinantes Sociales de la Salud. Ciudad de México: Editora Universidad Autónoma Metropolitana, 2011.
CABRERA, A. et al. Por una discusión acerca de la CDSS desde la perspectiva de la medicina social latinoamericana. In: EIBENSCHUTZ, C., TAMEZ, S, GONZÁLEZ, R. ¿Determinación social o determinantes sociales de la salud? Memoria del Taller Latinoamericanos sobre Determinantes Sociales de la Salud. Ciudad de México: Editora Universidad Autónoma Metropolitana, 2011.

CASSEL, J. The contribution of the Social environment to host resistance. American Journal of Epidemiology, London, v. 104, n. 2, 1976, p. 107-23.

\section{COMISSÃO PARA OS DETERMINANTES SOCIAIS} DA SAÚDE (CDSS). Redução das desigualdades no período de uma geração: igualdades na saúde através da ação sobre os determinantes sociais. Geneva: OMS, 2010.

EIBENSCHUTZ, C., TAMEZ, S., GONZÁLEZ, R. (Org.) ¿Determinación social o determinantes sociales de la salud? Memoria del Taller Latinoamericanos sobre Determinantes Sociales de la Salud. México, DF: Editora Universidad Autónoma Metropolitana, 2011.

GARCÍA, J. C. Pensamiento social en salud en América Latina. México, DF: OPAS: Intercamericana, 1994, p. 184-191.

HERNÁNDEZ-ÁLVAREZ, M. Desigualdad, inequidad e injusticia en el debate actul en salud: posiciones e implicaciones. In: EIBENSCHUTZ, C., TAMEZ, S., GONZÁLEZ, R. (Org.). ¿¿Determinación 
social o determinantes sociales? México, DF: Editora

Universidad Autónoma Metropolitana, 2011.

Neoliberalismo en salud: desarrollos, supuestos y alternativas. In: RESTREPO, D. (Org.). La falacia neoliberal. Crítica y alternativas. Bogotá: Editora Universidad Nacional de Colombia, 2003, p. 347-361.

INGLEBY, D. Ethnicity, Migration and the Social Determinants of Health Agenda. Psychosocial Intervention/Intervencion Psicosocial, Madrid, v. 21, n. 3, p. 331-341, 2012.

KAPLAN, G. A. et al. Inequality in income and mortality in the United States: analysis of mortality and potential pathways. British Medical Journal, London, v. 312, n. 7037,1996, p. 999-1003.

KRIEGER, N. Theories for social epidemiology in the 21st century: an ecosocial perspective. International Journal of Epidemiology, London, v. 30, n. 4, 2001, p. 668-77.

LANDER, E. (Org.) A colonialidade do saber: eurocentrismo e ciências sociais. Perspectivas latino-americanas. Colección Sur-Sur. Buenos Aires: CLACSO, 2005.

LAURELL, A. C. Para el estudio de la salud en su relación con el proceso de producción. In: ALAMES. Taller Latinoamericano de Medicina Social. Medellin: Editora Universidad de Antioquia, 1987, p. 61-94.

LYNCH, J. et al. Income inequality, the psychosocial environment, and health: comparisons of wealthy nations. Lancet, London, v. 358, n. 9277, p. 194-200, 2001

MARMOT, M.; WILKINSON, R. G. Psychosocial and material pathways in the relation between income and health: a response to Lynch et al. British Medical Journal, London, v. 322, n. 7296, p. 1233-6, 2001.

MORALES, C. et al. ¿¿Determinación social o determinantes sociales? Diferencias conceptuales e implicaciones praxiológicas. Rev. Salud Pública, Bogotá, v. 15 , n. 6 , p. $797-808,2013$

NAVARRO, V. What we mean by social determinants of health. International Journal of Health Services, Amityville, v. 39, n. 3, p. 423-441, 2009.

NOGUEIRA, R. P. (Org.) Determinação Social da Saúde e Reforma Sanitária. Rio de Janeiro: CEBES, 2010. (Coleção Pensar em Saúde).

PASSOS, R. Determinação Social da Saúde. Saúde em Debate, Rio de Janeiro, v. 33, n. 83, 2009.

PEÑARANDA, F.; OTÁLVARO, G. J. El debate determinantes-determinación social de la salud: aportes para la renovación de la praxis de la salud pública. Revista

Facultad Nacional de Salud Pública, Medellín, n. 31, 2013.

SANTOS, B. S. A gramática do tempo: Para uma nova cultura política. 3 ed. São Paulo: Cortez, 2010.

SMITH, G, D.; EGGER, M. Commentary: understanding it all--health, meta-theories, and mortality trends. British Medical Journal, London, v. 313, n. 584-5, 1996.

SOLAR, O.; IRWIN, A. A conceptual framework for action on the social determinants of health. Social Determinants of Health. Discussion Paper 2 (Policy and Practice). Geneva: WHO, 2010.

TAJER, D. Latin American social medicine: roots, development during the 1990s, and current challenges. American Journal of Public Health, Washington, DC, v. 93, n. 12, p. 2023-2027, 2003.

TAMBELLINI, A. T., SCHÜTZ, G. E. Contribuição para o debate do CEBES sobre determinação social da saúde: repensando processos sociais, determinações e determinantes da saúde. Saúde em Debate, Rio de Janeiro, v. 33, n. 83, p. 371-379, 2009.

WHITEHEAD, M.; DAHLGREN G. What can be done about inequalities in health? Lancet, London, v. 338, n. 8774, p. 1059-1063, 1991.

Recebido para publicação em outubro de 2014

Versão final em julho de 2015

Conflito de interesse: inexistente

Suporte financeiro: não houve 